\title{
Drug delivery to the brain by focused ultrasound induced Blood-Brain Barrier Disruption: Quantitative Evaluation of Enhanced Permeability of Cerebral Vasculature Using Two-Photon Microscopy
}

T. Nhan, A. Burgess, E. Cho, B. Stefanovic, L. Lilge, K. Hynynen

Version Post-print/accepted manuscript

Citation T. Nhan, A. Burgess, E. Cho, B. Stefanovic, L. Lilge, K. Hynynen, (published version) "Drug delivery to the brain by focused ultrasound induced Blood-Brain Barrier Disruption: Quantitative Evaluation of Enhanced Permeability of Cerebral Vasculature Using Two-Photon Microscopy", Journal of Controlled Release, 2013 Nov 28;172(1):274-80. doi: 10.1016/j.jconrel.2013.08.029

Additional publisher The final published version of this article is available at information http://dx.doi.org/10.1016/j.jconrel.2013.08.029.

Copyright/License (c) (i) $\odot$ This work is licensed under the Creative Commons International License. To view a copy of this license, visit http://creativecommons.org/licenses/by-nc-nd/4.0/.

How to cite TSpace items

Always cite the published version, so the author(s) will receive recognition through services that track citation counts, e.g. Scopus. If you need to cite the page number of the author manuscript from TSpace because you cannot access the published version, then cite the TSpace version in addition to the published version using the permanent URI (handle) found on the record page.

This article was made openly accessible by $U$ of $T$ Faculty. Please tell us how this access benefits you. Your story matters. 


\title{
Drug Delivery to the Brain by Focused Ultrasound Induced Blood-Brain Barrier Disruption: Quantitative Evaluation of Enhanced Permeability of Cerebral Vasculature Using Two- Photon Microscopy
}

\author{
Tam Nhan ${ }^{a, b}$, Alison Burgess ${ }^{a}$, Eunice E. Cho ${ }^{a}$, Bojana Stefanovic ${ }^{a, b}$, Lothar Lilge ${ }^{b, c}$, and \\ Kullervo Hynynen ${ }^{a, b}$ \\ aPhysical Sciences, Sunnybrook Research Institute, Toronto, ON Canada \\ bMedical Biophysics, University of Toronto, Toronto, ON Canada \\ 'Ontario Cancer Institute, Princess Margaret Hospital, Toronto, ON Canada
}

\begin{abstract}
Reversible and localized blood-brain barrier disruption (BBBD) using focused ultrasound (FUS) in combination with intravascularly administered microbubbles (MBs) has been established as a non-invasive method for drug delivery to the brain. Using two-photon fluorescence microscopy (2PFM), we imaged the cerebral vasculature during BBBD and observed the extravasation of fluorescent dye in real-time in vivo. We measured the enhanced permeability upon BBBD for both $10 \mathrm{kDa}$ and $70 \mathrm{kDa}$ dextran conjugated Texas Red (TR) at the acoustic pressure range of 0.2-0.8 MPa and found permeability constants of TR10kDa and TR70kDa vary from 0.0006 to 0.0359 $\mathrm{min}^{-1}$ and 0.0003 to $0.0231 \mathrm{~min}^{-1}$, respectively. For both substances, a linear regression was applied on the permeability constant against the acoustic pressure and the slope from best-fit was found to be $0.039 \pm 0.005 \mathrm{~min}^{-1} / \mathrm{MPa}$ and $0.018 \pm 0.005 \mathrm{~min}^{-1} / \mathrm{MPa}$, respectively. In addition, the pressure threshold for successfully induced BBBD was confirmed to be 0.4-0.6 MPa. Finally, we identified two types of leakage kinetics (fast and slow) that exhibit distinct permeability constants and temporal disruption onsets, as well as demonstrated their correlations with the applied acoustic pressure and vessel diameter. Direct assessment of vascular permeability and insights on its dependency on acoustic pressure, vessel size and leakage kinetics are important for treatment strategies of BBBD-based drug delivery.
\end{abstract}

\section{Keywords}

Blood-brain barrier; focused ultrasound; drug delivery; permeability; two-photon fluorescence microscopy

\footnotetext{
(C) 2013 Elsevier B.V. All rights reserved.

Corresponding Author: Tam Nhan, Physical Sciences, Sunnybrook Research Institute, C713 - 2075 Bayview Ave, Toronto, ON M4N 3M5, Canada, Phone: 289-200-7245, Fax: 416-480-5714, tnhan@ sri.utoronto.ca.

Publisher's Disclaimer: This is a PDF file of an unedited manuscript that has been accepted for publication. As a service to our customers we are providing this early version of the manuscript. The manuscript will undergo copyediting, typesetting, and review of the resulting proof before it is published in its final citable form. Please note that during the production process errors may be discovered which could affect the content, and all legal disclaimers that apply to the journal pertain.
} 


\section{Introduction}

Intensive efforts in drug development have led to the formation of numerous therapeutics with the potential to treat central nervous system (CNS) diseases and disorders including chemotherapeutic agents for the treatment of brain tumours and metastases [1] as well as chemokines, growth factors, and viral vectors for the treatment of neurodegenerative diseases [2-4]. However, the blood-brain barrier (BBB) prevents more than $98 \%$ of the existing pharmaceutical agents from entering the brain tissue $[5,6]$ thereby limiting the application of these potential therapeutics in the CNS. Research efforts are underway to develop clinically viable methods to deliver therapeutic drugs into the CNS safely and effectively such as drug modification to promote carrier-mediated or receptor-mediated transport $[7,8]$ as well as intra-carotid infusion of hyper-osmotic solutions to cause the widening of tight junctions $[9,10]$.

Recently, focused ultrasound (FUS) in combination with microbubble (MB) contrast agent, have been used as a non-invasive method to induce BBB disruption (BBBD) for spatially targeted drug delivery to the brain [11]. Inside the blood vessel, under the influence of the FUS beam, MBs expand and contract via inertial cavitation or stable cavitation processes [12-15]. These physical activities exert mechanical forces (i.e. shear and circumferential stresses) onto proximal capillary walls [16] leading to increased paracellular transport through widened tight junctions [17], and/or increased transcellular transport through activation of carrier proteins or pore formation [18-21]. In general, localization of the BBB opening is dictated by the focal volume of the FUS beam and the presence of MBs which reduce the ultrasound energy required for BBBD so that thermal effects and tissue damage can be minimized. Using FUS, effective drug concentrations can be achieved locally while their accumulation elsewhere in the brain, and body can be limited, hence decreasing nonspecific effects.

Guidance and evaluation of FUS-induced BBBD has been primarily performed by magnetic resonance imaging (MRI). Typically, T1-weighted MR images are acquired to confirm successful delivery of Gadolinium based MR contrast agents across the BBB [11,22,23], whereas T2-weighted MR images serve to verify the absence of edema and tissue damage $[24,25]$. In addition, quantitative measurement of the permeability of the BBB in the targeted region (e.g. hippocampus or striatum) can be done via dynamic contrast enhanced MRI (DCE-MRI) [26-29]. However, spatial resolution (e.g. lateral: $86 \times 86 \mu \mathrm{m}^{2}$; slice thickness: $500 \mu \mathrm{m}$ ) offered by MRI limits permeability measurement to a macroscopic brain volume of $2-35 \mathrm{~mm}^{3}[28,29]$.

To advance knowledge of the microscopic mechanisms associated with FUS-induced $\mathrm{BBBD}$, we proposed using two-photon fluorescence microscopy (2PFM) which allows sufficient temporal and spatial resolution to track transient changes in BBB permeability at a microvascular level. Beyond volumetric average as achieved in DCE-MRI, we hope to resolve the gradient of drug concentration to differentiate therapeutic range from toxicity level using 2PFM. Proof-of-concept in monitoring BBBD using 2PFM had been demonstrated by Raymond et al. [30,31]. In that study, the authors injected fluorescent dyes (e.g. Texas Red, Oregon Green) for the visualization of the microvasculature and transmitted ultrasound from the ventral surface of the brain to induce BBBD. With this setup, the authors identified two leakage responses: micro-disruption and slow disruption. The former is characterized by a focal eruption of dye from a single point on the vessel wall, whereas the latter is characterized by a slower and more spatially-extended leakage. Using a dorsal approach for application of FUS, our group was able to reliably induce BBBD with a bettercontrolled in situ applied, acoustic pressure [32,33]. These studies characterized the 
microscopic leakage patterns qualitatively but did not attempt to quantify the rate of agent delivery.

Here, we demonstrate a quantitative approach to analyze the 2PFM images post BBBD. By extracting and correlating intravascular and extravascular signals from the time-lapse 2PFM images, a permeability constant of the cerebral vasculature network within the imaging field of view (FOV) can be determined. Measured vascular permeability is then correlated with the applied acoustic pressure, disruption onset and vessel diameter to shed light on the potential mechanisms which control BBBD. These insights are crucial for guiding future treatments utilizing BBBD-based drug delivery to the brain.

\section{Material and Methods}

\section{Animal preparation}

Male Wistar rats of $150-250 \mathrm{~g}$ weight range were used in this study $(\mathrm{n}=40)$. All the procedures were approved by the institutional Animal Care and Use Committee and were in accordance with the guideline by Canadian Council on Animal Care. The animals were initially anesthetized with $5 \%$ isofluorane, which was later reduced to $2 \%$. The animals were positioned in a stereotactic frame and the head was immobilized using ear and bite bars (Figure 1A). The skull and dura were removed to create a $5 \mathrm{~mm}$ diameter cranial window over the right hemisphere, approximately $3 \mathrm{~mm}$ lateral from the midline and $3 \mathrm{~mm}$ posterior to the bregma. The cranial window was filled with $1 \%$ agarose and the transducer attached to a coverslip of $12 \mathrm{~mm}$ diameter and $150 \mu \mathrm{m}$ thickness was secured on top of the cranial window by cyanoacrylate glue (Figure 1A). The tail vein was cannulated with a $23-\mathrm{G}$ needle and $70 \mathrm{~mm}$ polyethylene tubing for the, injection of fluorescent dye and MB contrast agents. Once the surgery was completed, the stereotactic stage was transferred to the microscope for BBBD induction and 2PFM imaging.

\section{FUS parameters for BBBD}

The experimental timeline is shown in Figure 1B. Prior to sonication, Definity MBs (Lantheus Medical Imaging, Billerica, MA, USA) of 1.1-3.3 $\mu \mathrm{m}$ mean diameter were diluted with saline $(1: 10 \mathrm{v} / \mathrm{v})$ and injected through the tail vein at a final concentration of $0.02 \mathrm{ml} /$ $\mathrm{kg}$. A PZT-4 cylindrical transducer (diameter $=10 \mathrm{~mm}$, thickness $=1.5 \mathrm{~mm}$, height $=1.1$ $\mathrm{mm}$ ) was used for sonication. A complete characterization study of the transducer design, which facilitates dorsal application of FUS and simultaneous 2PFM imaging, has been described previously in [33]. Briefly, the transducer was operated in the thickness mode at a frequency of $1.2 \mathrm{MHz}$ to produce a circularly uniform focal spot that coincides with the microscope's lateral imaging FOV $\left(512 \times 512 \mu \mathrm{m}^{2}\right)$. The ultrasound depth of field generated by the transducer is shallow ( $1 \mathrm{~mm}$ immediately beneath the coverslip), ensuring that it overlapped with the light depth penetration of the $2 \mathrm{PFM}$. The transducer was driven by a function generator (Agilent, Palo Alto, CA, USA) and a $53 \mathrm{~dB}$ RF power amplifier (NP Technologies Inc., Newbury Park, CA, USA) with typical BBBD sonication parameters (10 ms pulse duration, $1 \mathrm{~Hz}$ pulse repetition frequency, $120 \mathrm{~s}$ total sonication duration). The applied forward and reflected RF-power during sonication was recorded using an in-house constructed power meter.

\section{PFM imaging}

To visualize the cerebral vasculature, $10 \mathrm{kDa}$ or $70 \mathrm{kDa}$ dextran-conjugated Texas Red (Invitrogen, Burlington, ON, Canada) was injected through the tail vein. The two molecular weights (MWs) were chosen to represent equivalent therapeutic substances with sizes ranging from small proteins and siRNAs up to albumin-bound drugs that can be delivered across the BBB. The animal was positioned below the microscope stage (FV1000MPE, 
Olympus, Tokyo, Japan) and the cranial window was aligned underneath, a waterimmersion objective (Olympus XLPLN, Tokyo, Japan) with 25× magnification power, 1.05 numerical aperture and $2 \mathrm{~mm}$ working distance. Two-photon excitation of Texas Red fluorescent dye was achieved with a mode-lock Ti:Sapphire laser unit (Mai-Tai, SpectraPhysics, Mountain View, CA, USA) emitting at $810 \mathrm{~nm}$ wavelength, $100 \mathrm{fs}$ pulse width and $80 \mathrm{MHz}$ repetition rate. Scanning was performed in an XYZT order (Figure 1C), in which lateral images of $512 \times 512$ pixels $(0.99 \mu \mathrm{m}$ resolution, $8 \mu \mathrm{s} /$ pixel $)$ were captured below the cortical surface up to $300 \mu \mathrm{m}$ depth (i.e. cortical layers I and II) in a stacking mode at $10 \mu \mathrm{m}$ increments. This stacking distance allows imaging of pial vessels as well as penetrating vessels and capillaries. As depicted in Figure 1B, 2PFM imaging was continuous over the course of the experiment; from the injection of MBs, through the $120 \mathrm{~s}$ sonication, and following leakage of the fluorescent dye upon BBBD. Typically, each data set consists of 40-50 stacks with the acquisition time of 15 to 30 seconds per stack.

\section{Analysis of 2PFM data}

4D XYZT microscopic data of a superficial cortical tissue volume was visualized in Matlab (The Mathworks, Natick, MA, USA) as a maximum intensity projection map along $\mathrm{z}$ direction (Figure 2A). To separate the intravascular and extravascular compartment, automatic vessel segmentation was performed on each individual Z-slice at the initial time point (when BBB was impermeable to either dextran). Once the intravascular regions of interest (ROIs) were masked based on the segmented vessels, extravascular ROIs were identified by subtracting the intravascular ROIs from the imaging FOV. Fluorescent intensity associated with each compartment, $\mathrm{I}_{\mathrm{i}}(\mathrm{t})$ and $\mathrm{I}_{\mathrm{e}}(\mathrm{t})$, was then calculated by averaging over all pixels within the compartment ROIs over the entire depth (Figure 2B).

To measure permeability from fluorescent intensity change in the intravascular and extravascular space, we applied the formulation developed by Dreher et al. [34]. In their model, the rate of solute transport across a blood vessel wall is given by the KedemKatchalsky equation to account for both convection and diffusion processes [34-36]. However, due to its unknown direction and magnitude, the convective term was ignored and its influence was lumped into the latter term [37]. Given the linearity between the dye concentration and the fluorescent signal intensity in the plasma and the extravascular space, an apparent permeability $a(t)$ measuring exchange capacity between the two compartments can be determined via the following equation:

$$
\alpha(t)=\frac{d I_{e} / d t}{\frac{I_{i}(t)}{1-H C T}-\frac{I_{e}(t)}{V_{e} / V_{i}}}
$$

where $\mathrm{V}_{\mathrm{e}} / \mathrm{V}_{\mathrm{i}}$ is the volume fraction between extravascular and intravascular compartments, which was simply obtained from the vessel segmentation. Similar to DCE-MRI studies by Park et al. [27] and Vlachos et al. [28,29], HCT of 45\% was assigned to account for the average hematocrit level of all blood vessels within the imaging FOV [38,39]. Apparent permeability $a_{\text {app }}$ for each studied subject is the average value of $a(t)$ from 3 to 30 minutes, corresponding to the peaked duration of BBBD (Figure 2C).

\section{Statistical analysis}

At each acoustic pressure, the apparent permeability $a_{\text {app }}$ of TR10kDa and TR70kDa, as well as the volume fraction $\mathrm{V}_{\mathrm{i}} / \mathrm{V}_{\mathrm{e}}$, were reported as mean ( \pm standard deviation) over 5 animal subjects. For 40 cases of permeability measurements, comparison among 4 pressure groups and 2 molecular weights ( $\mathrm{MW}=10 \mathrm{kDa}$ and, $70 \mathrm{kDa}$ ) was performed using two-way 
ANOVA followed by Bonferroni post-tests in GraphPad Prism (GraphPad Software Inc., CA, USA). Correlation between the acoustic pressure and BBBD temporal onset was evaluated by one-way ANOVA followed by Bonferroni's Multiple Comparison Test. Distinction between vessel size distribution and leakage type (fast vs. slow kinetics) was assessed using two-tailed unpaired Student's $t$ test. For all of these analyses, $p<0.05$ were considered statistically significant.

\section{Results \\ Effect of acoustic pressure on enhanced BBB permeability}

For TR10kDa and TR70kDa, we analyzed 5 data sets per pressure and the evaluated permeability values are presented in Figure 3A and Figure 3B, respectively. Both scattering plots reveal an increasing trend for the permeability at higher pressure. To quantitatively explore the relationship of permeability and acoustic pressure, linear regression was applied for each MW. Best-fit values of slope for TR10kDa and TR70kDa were found to be $0.039 \pm 0.005 \mathrm{~min}^{-1} / \mathrm{MPa}$ and $0.018 \pm 0.005 \mathrm{~min}^{-1} / \mathrm{MPa}$, respectively. Best-fit values of Xintercept for TR10kDa and TR70kDa were found to be $0.16 \mathrm{MPa}$ and $0.11 \mathrm{MPa}$, respectively. In addition, two-way ANOVA in combination with Bonferroni post-tests confirmed statistical significance in permeability of TR10kDa induced at low acoustic pressure of $0.4 \mathrm{MPa}$ with respect to higher acoustic pressure of $0.6 \mathrm{MPa}$ and $0.8 \mathrm{MPa}$. For TR70kDa, no significant difference of permeability between $0.4 \mathrm{MPa}$ and $0.6 \mathrm{MPa}$ was found, whereas statistical significance was observed for resulting permeabilities at $0.4 \mathrm{MPa}$ and $0.8 \mathrm{MPa}$.

\section{Effect of substance size on enhanced BBB permeability}

To determine the impact of substance size (or MW) on BBB permeability, average values of two MWs at similar acoustic pressure are plotted side by side as shown in Figure 4A. Twoway ANOVA followed by Bonferroni post-tests confirmed that the difference in enhanced permeability between the two MWs at $0.2 \mathrm{MPa}$ and $0.4 \mathrm{MPa}$ was not significant. However, at high pressure, a significantly greater permeability of TR10kDa compared to TR70kDa was demonstrated (e.g. $p<0.05$ at $0.6 \mathrm{MPa}$ and $0.8 \mathrm{MPa}$ ), which is consistent with the expected inverse relationship between molecular size and its permeation across the BBB. To confirm that these permeability measurements were not confounded by differences in cerebral vasculature volume, two-way ANOVA followed by Bonferroni post-tests were performed on the volume fraction $\mathrm{V}_{\mathrm{i}} / \mathrm{V}_{\mathrm{e}}$ between TR10kDa and TR70kDa at 4 acoustic pressure levels ( $\mathrm{n}=5$ per group) and found no statistically significant differences for all 4 pairs.

\section{Temporal onset of BBBD is correlated with permeability and appears to be controlled by acoustic pressure}

To investigate the kinetics of BBBD, temporal onset (i.e. time point when leakage was initiated) was recorded for all 40 data sets and plotted against the permeability. As evident in Figure 5A, an inverse relationship between these two entities was observed. 10 minutes was chosen as the temporal benchmark to separate fast leakage from slow leakage as this duration is the averaged time required for receptor-mediated transcytosis [39,40]. Fast leakage exhibits short BBBD onset and substantially higher permeability constants, whereas slow leakage presents delayed BBBD onset and very small permeability.

To examine the connection between BBBD onset and the applied acoustic pressure, averaged temporal onset was plotted at each pressure $(n=10$ per pressure), as shown in Figure 5B. Due to its gradual increase in leakage, it is difficult to define BBBD onset time based on the extravascular signal curve (e.g. Figure 2B). Therefore, we used the peak time 
of the permeability curve (e.g. Figure 2C) as the benchmark for BBBD onset time. In doing so, we further noted that the peak time of approximately 4-5 minutes agrees with visible leakage evidence of Texas Red extravasating out of initially intact vasculature as shown in Figure 2A.

Overall, higher pressure appears to yield prompt onset. One-way ANOVA followed by Bonferroni's Multiple Comparison Test reported no statistical significance between 0.2-0.4 $\mathrm{MPa}$ and 0.6-0.8 MPa, whereas significant difference between average BBBD onset at 0.4 $\mathrm{MPa}(13.5 \pm 7.6$ minutes) and at $0.6 \mathrm{MPa}(6.3 \pm 4.3$ minutes) was noted. This strongly suggests that different leakage kinetics (slow or fast) can be controlled via the applied acoustic pressure.

\section{Effect of vessel diameter on enhanced BBB permeability}

We further looked into the effect of vessel diameter on the enhancement of BBB permeability by measuring the average diameter of vessels undergoing disruption within the imaging FOV and plotting the value against the corresponding permeability constant as illustrated in Figure 6A. Overall, permeability appears to be inversely related to vessel diameter. By applying the predefined criteria (10 minutes benchmark of BBBD onset and negligible permeability constant), data points associated with fast and slow leakage are separated by the dotted line in Figure 6A. Here, we noted that fast leakage is prevalent in small vessels (10-30 $\mu \mathrm{m}$ diameter), whereas slow leakage occurs more commonly in larger vessels (30-70 $\mu \mathrm{m}$ diameter). As revealed in Figure 6B, $p$ value, from two-tailed unpaired Student's $t$ test confirms the statistical significance in vessel size distribution with respect to leakage type.

\section{Discussion}

Past investigations into the kinetics of BBBD permeability had been carried out using DCEMRI. For instance, Vlachos et al. demonstrated the reconstructed permeability map of the murine hippocampus superimposed onto coronal and transverse T1 images of the brain $[28,29]$. The same group also confirmed the dependence of permeability on acoustic pressures and microbubble sizes. Park et al. further compared the permeability enhancement of double sonication to single sonication. These analyses supported the use of DCE-MRI as an in vivo tool for quantifying the efficacy of FUS induced BBB opening. However, detecting disruption and measuring permeability at vascular level is of fundamental importance for resolving concentration gradients of the delivered drugs, from which therapeutic range and excessive toxicity range can both be identified. Furthermore, permeability provides estimation of drug concentration in the interstitial space. To perform these analyses, 2PFM is required.

Our study builds on the initial work of Raymond et al. who demonstrated the use of 2PFM for a comprehensive investigation of BBBD [31]. Our previously published study has advanced this field by introducing dorsally applied FUS which enhances the robustness of BBBD at a reliable in situ pressure [32,33]. In the present work, we presented a quantitative analysis technique which allows for characterization of permeability from 2PFM timelapsed images. High spatial resolution from 2PFM imaging enabled discerning investigation into vessel diameter and the temporal onset of BBBD in correlation with applied acoustic pressure and resulting disruption kinetics.

Permeability constants had been previously reported for $1 \mathrm{kDa}$ Gadolinium diethylenetriamine penta-acetic acid (Gd-DTPA) via DCE-MRI measurement as 1.1e-2 $\mathrm{min}^{-1}$ and 3.9e-2 $\mathrm{min}^{-1}$ when BBBD was achieved at frequency of $1.5 \mathrm{MHz}$ and acoustic, pressure of $0.45 \mathrm{MPa}$ and $0.6 \mathrm{MPa}$, respectively [29]. At comparable FUS parameters, these 
values are two-fold and five-fold higher than 2PFM monitored BBB permeability enhancement of TR10kDa and TR70kDa, respectively. Although differences in permeability are expected due to its ten-fold smaller size, the distinctions between the two imaging modalities should be noted. In 2 PFM, a small cortical tissue volume $\left(512 \times 512 \times 100 \mu^{3}\right)$ is directly measured and vessels of narrow size distribution undergoing BBBD are readily detected. In contrast, value reported from DCE-MRI is corresponding to a much larger brain tissue volume (2-35 $\left.\mathrm{mm}^{3}\right)$ [27-29] that contains only a fraction of vessels at broader size distribution being disrupted while the rest of vascular tree remain intact. Nevertheless, these data fit the overall expected trend of an inverse correlation between MW and permeability constant and eliminates the need to conduct further 2PFM on small MW compounds.

Furthermore, we speculate that the permeability of large molecule therapeutics would follow the same trend as seen here and thus exhibit lower permeability than the $70 \mathrm{kDa}$ compound. However, one limitation of this study is that we cannot predict how drugs with charges or other modifications will extravasate upon BBBD. Similarly, stem cells and immune cells have been shown to cross the BBB upon the application of FUS+MBs [41,42]. Due to the ability of the cells to interact with the BBB, understanding the kinetics may require further analysis.

From this study, we also noted that the permeability constant is linearly related to the applied acoustic pressure, as well as inversely related to the onset of BBBD. This observation can be explained by higher acoustic pressure resulting in greater oscillatory amplitude of MBs that to induce vascular effects, and/or inertial cavitation, which triggers the collapse of MBs [12]. These MB activities could readily prompt the opening, of BBB. We also observed that transition from low to high permeability (Figure 3), as well as from long to short BBBD onset (Figure 5B) takes place at the pressure range of 0.4-0.6 MPa. This transitional pressure agrees well with the expected pressure of $0.5 \mathrm{MPa}$ when considering the operating frequency of $1.2 \mathrm{MHz}$ used in our 2PFM-guided experiment and the mechanical index for BBBD threshold of 0.46 [43].

By classifying the leakage types into fast and slow kinetics based on BBBD onset (Figure $5 \mathrm{~A}$ ), we found that 20 of 22 data points in the first group exhibiting high permeability (from $0.005 \mathrm{~min}^{-1}$ up to $0.036 \mathrm{~min}^{-1}$ ). On the other hand, in the latter group, 16 of 18 data points possess permeability constants below $0.005 \mathrm{~min}^{-1}$. Mechanistically, we speculate that the fast leakage is caused by the opening tight junctions, leading to fluorescent dye leakage out of the blood vessel. In some cases, $\mathrm{I}_{\mathrm{e}}(\mathrm{t})$ curve exhibits saturation after the initial ramp-up (15-20 minutes post sonication), indicating quick repair and closure of the BBB of a few vessels in the imaging FOV. One study suggested that a possible mechanism for quick repair involves the recruitment of astrocytes and microglia to the disruption site [44]. Although opening of tight junctions might be responsible for the typical focal pattern of disruption, another possible mechanism of fast leakage is cellular sonoporation [19]. This might explain why some fast leakage occurred extensively along a segment of blood vessel rather than from a single focal point. Notably for this mechanism, the pores also reseal quickly (4-10 seconds) [45]. In contrast, we predict that slow leakage is facilitated by transcytosis, which is limited under normal condition of the BBB. However, FUS-induced oscillation of MBs may activate endothelial cell receptors to promote transcellular transport of molecules from the lumen to interstitial space $[18,21,46]$. It was previously suggested that transcytosis of low-density lipoproteins across the endothelial cells lining the vessel, wall takes at least 15 minutes [39]. This lag may account for the slow onset of leakage observed in our 2PFM experiments.

As reflected in the permeability constant, the extent of BBB opening is inversely related to the vessel diameter (Figure 6A). In a simulation study, Hosseinkhah et al. had shown similar dependency of shear stress on the ratio between vessel and initial bubble radii (i.e., largest 
shear stresses were obtained at lowest $r_{v} / r_{0}$ values) [16]. Based on this data, we speculate that when MBs with a narrow size distribution are administered, smaller vessels will experience higher shear stress and be more prone to BBB opening. This conjecture also describes the observed link between vessel size and leakage type (shown in Figure 6B), where smaller vessels are more inclined to undergo fast leakage due to the applied shear stress, while slow leakage is predominately seen in larger vessels under trigger by minor perturbations and activation of cellular receptors. Furthermore, this notion is in agreement with electron microscopy observations where higher level of active vesicular transport of blood-borne tracer molecules was found in the arterioles as compared to the capillaries [21].

Lastly, the enhanced permeability, time of BBBD onset, and leakage kinetics (fast vs. slow) are affected by the applied acoustic pressure. This suggests that it is possible to control the leakage type and tailor drug delivery for specific treatment procedures by altering the FUS parameters. For instance, the high permeability and prompt opening associated with fast leakage may benefit delivery of small MW drugs with short plasma half-lives. In contrast, slow leakage may be more suitable for delivery of large MW substances, with increased plasma half-lives that allows for extended availability for transcytocis across the BBB. In fact, by considering the difference in plasma half-lives between $10 \mathrm{kDa}$ and $70 \mathrm{kDa}$ agent (e.g. 10 minutes vs. 25 minutes [34]) and their, averaged permeability constant associated with fast leakage (e.g. $0.0205 \mathrm{~min}^{-1}$ vs. $0.0110 \mathrm{~min}^{-1}$, respectively (Figure 6A)), we calculated the accumulation of each agent delivered to the extravascular compartment over 1 hour. As a result, the fraction of concentration-time area under the curve (AUC) between extravascular space and plasma was estimated for $10 \mathrm{kDa}$ and $70 \mathrm{kDa}$ agent to be 0.61 and 0.35 , respectively. Meanwhile, given a low averaged permeability constant of $0.0025 \mathrm{~min}^{-1}$ for both MWs (Figure 6A), the fraction of concentration-time AUC between the two compartments was found to be relatively comparable (e.g. 0.11 for $10 \mathrm{kDa}$ and 0.09 for 70 $\mathrm{kDa}$ ). Therefore, this quantitative approximation supports the aforementioned postulations on suitable delivery approaches for therapeutic agent of different MWs (e.g. fast leakage for small MWs and slow leakage for large MWs).

\section{Acknowledgments}

The authors would like to thank Shawna Rideout-Gros and Alexandra Garces for their help with animal care; Adrienne Dorr for her guidance in using the two-photon fluorescence microscope for our in vivo studies; and Shirley Miao for her assistance in developing Matlab vessel segmentation algorithm. This work was supported in part by National Institutes of Health under Grant \# R01 EB003268 (Kullervo Hynynen), Canadian Institutes of Health Research under Grant \# CIHR FRN 119312 (Kullervo Hynynen) and Natural Sciences and Engineering Research Council of Canada under NSERC CGS-D3 Scholarship (Tam Nhan).

\section{References}

1. Huse JT, Holland EC. Targeting brain cancer: advances in the molecular, pathology of malignant glioma and medulloblastoma. Nature Reviews Cancer. 2010; 10:319-31.

2. Weiner HL, Frenkel D. Immunology and immunotherapy of Alzheimer's disease. Nature Reviews Immunology. 2006; 6:404-16.

3. Tuszynski MH. Growth-factor gene therapy for neurodegenerative disorders. The Lancet Review. 2002; 1:51-57.

4. Lim ST, Airavaara M, Harvey BK. Viral vectors for neurotrophic factor, delivery: a gene therapy approach for neurodegenerative diseases of the CNS. Pharmacological Research. 2010; 61:14-26. [PubMed: 19840853]

5. Goldstein GW, Betz AL. The blood-brain barrier. Scientific American. 1986; 255:74-83. [PubMed: 3749857]

6. Pardridge WM. The blood-brain barrier: bottleneck in brain drug development. NeuroRx. 2005; 2:3-14. [PubMed: 15717053] 
7. Nies AT. The role of membrane transporters in drug delivery to brain tumors. Cancer Letters. 2007; 254:11-29. [PubMed: 17275180]

8. Juillerat-Jeanneret $\mathrm{L}$. The targeted delivery of cancer drugs across the blood-brain barrier: chemical modifications of drugs or drug-nanoparticles? Drug Discovery Today. 2008; 13:1099-106. [PubMed: 18848640]

9. Rapoport SI. Advances in osmotic opening of the blood-brain barrier to enhance, CNS chemotherapy. Expert Opinion on Investigational Drugs. 2001; 10:1809-18. [PubMed: 11772287]

10. Kraemer DF, Fortin D, Neuwelt EA. Chemotherapeutic dose intensification for treatment of malignant brain tumors: recent developments and future directions. Current Neurology and Neuroscience Reports. 2002; 2:216-24. [PubMed: 11937000]

11. Hynynen K, McDannold N, Vykhodtseva N, Jolesz FA. Noninvasive MR Imaging - guided Focal Opening of the Blood-Brain Barrier in Rabbits. Radiology. 2001; 220:640-646. [PubMed: 11526261]

12. McDannold N, Vykhodtseva N, Hynynen K. Targeted disruption of the blood-brain barrier with focused ultrasound: association with cavitation activity. Physics in Medicine and Biology. 2006; 51:793-807. [PubMed: 16467579]

13. O'Reilly MA, Hynynen K. Feedback-controlled Focused Ultrasound Disruption by Using an Acoustic Emissions - based Controller. Radiology. 2012; 263

14. O'Reilly MA, Hynynen K. Ultrasound enhanced drug delivery to the brain and central nervous system. International Journal of Hyperthermia. 2012; 28:386-96. [PubMed: 22621739]

15. Burgess A, Hynynen K. Noninvasive and Targeted Drug Delivery to the Brain Using Focused Ultrasound. ACS Chemical Neuroscience. 2013

16. Hosseinkhah N, Hynynen K. A three-dimensional model of an ultrasound, contrast agent gas bubble and its mechanical effects on microvessels. Physics in Medicine and Biology. 2012; 57:785-808. [PubMed: 22252221]

17. Sheikov N, McDannold N, Sharma S, Hynynen K. Effect of focused, ultrasound applied with an ultrasound contrast agent on the tight junctional integrity of the brain microvascular endothelium. Ultrasound in Medicine \& Biology. 2008; 34:1093-104. [PubMed: 18378064]

18. Deng J, Huang Q, Wang F, Liu Y, Wang Z, Wang Z, et al. The Role of, Caveolin-1 in Blood-Brain Barrier Disruption Induced by Focused Ultrasound Combined with Microbubbles. Journal of Molecular Neuroscience. 2011

19. van Wamel A, Kooiman K, Harteveld M, Emmer M, ten Cate FJ, Versluis M, et al. Vibrating microbubbles poking individual cells: drug transfer into cells via sonoporation. Journal of Controlled Release. 2006; 112:149-55. [PubMed: 16556469]

20. Meijering BDM, Juffermans LJM, van Wamel A, Henning RH, Zuhorn IS, Emmer M, et al. Ultrasound and microbubble-targeted delivery of, macromolecules is regulated by induction of endocytosis and pore formation. Circulation Research. 2009; 104:679-87. [PubMed: 19168443]

21. Sheikov N, McDannold N, Jolesz F, Zhang YZ, Tam K, Hynynen K. Brain arterioles show more active vesicular transport of blood-borne tracer molecules than capillaries and venules after focused ultrasound-evoked opening of the blood-brain barrier. Ultrasound in Medicine \& Biology. 2006; 32:1399-409. [PubMed: 16965980]

22. McDannold N, Vykhodtseva N, Raymond S, a Jolesz F, Hynynen K. MRI-guided targeted bloodbrain barrier disruption with focused ultrasound: histological findings in rabbits. Ultrasound in Medicine \& Biology. 2005; 31:1527-37. [PubMed: 16286030]

23. Choi JJ, Pernot M, Small SA, Konofagou EE. Noninvasive, transcranial and localized opening of the blood-brain barrier using focused ultrasound in mice. Ultrasound in Medicine \& Biology. 2007; 33:95-104. [PubMed: 17189051]

24. O'Reilly M, Waspe AC, Ganguly M, Hynynen K. Focused-ultrasound, disruption of the bloodbrain barrier using closely-timed short pulses: influence of sonication parameters and injection rate. Ultrasound in Medicine \& Biology. 2011; 37:587-94. [PubMed: 21376455]

25. McDannold N, Vykhodtseva N, a Jolesz F, Hynynen K. MRI investigation of the threshold for thermally induced blood-brain barrier disruption and brain tissue damage in the rabbit brain. Magnetic Resonance in Medicine. 2004; 51:913-23. [PubMed: 15122673] 
26. Tofts PS, Brix G, Buckley DL, Evelhoch JL, Henderson E, Knopp MV, et al. Estimating Kinetic Parameters from Dynamic Contrast-Enhanced T1-Weighted MRI of a Diffusable Tracer: Standardized Quantities and Symbols. Magnetic Resonance Imaging. 1999; 232:223-232.

27. Park J, Zhang Y, Vykhodtseva N, a Jolesz F, McDannold NJ. The kinetics of blood brain barrier permeability and targeted doxorubicin delivery into brain induced by focused ultrasound. Journal of Controlled Release. 2012; 162:134-42. [PubMed: 22709590]

28. Vlachos F, Tung YS, Konofagou EE. Permeability assessment of the focused ultrasound-induced blood-brain barrier opening using dynamic contrast-enhanced MRI. Physics in Medicine and Biology. 2010; 55:5451-66. [PubMed: 20736501]

29. Vlachos F, Tung YS, Konofagou E. Permeability dependence study of the, focused ultrasoundinduced blood-brain barrier opening at distinct pressures and microbubble diameters using DCEMRI. Magnetic Resonance in Medicine. 2011; 830:821-830. [PubMed: 21465543]

30. Raymond SB, Skoch J, Bacskai BJ. A Ultrasound, Modular Design for In Vivo Optical Imaging and Ultrasound Treatment in the Murine Brain, IEEE Transactions on Ultrasonics Ferroelectrics, and Frequency Control. 2007; 54:431-434.

31. Raymond SB, Skoch J, Hynynen K, Bacskai BJ. Multiphoton imaging of, ultrasound/Optison mediated cerebrovascular effects in vivo. Journal of Cerebral Blood Flow and Metabolism. 2007; 27:393-403. [PubMed: 16685254]

32. Cho EE, Drazic J, Ganguly M, Stefanovic B, Hynynen K. Two-photon fluorescence microscopy study of cerebrovascular dynamics in ultrasound-induced blood-brain barrier opening. Journal of Cerebral Blood Flow and Metabolism. 2011:1-11.

33. Nhan T, Burgess A, Hynynen K. Transducer Design and Characterization for Dorsal Based Ultrasound Exposure and Two Photon Imaging of in vivo Blood-Brain Barrier Disruption in a Rat Model, IEEE Transactions on Ultrasonics. Ferroelectrics and Frequency Control. 2013 In Press.

34. Dreher MR, Liu W, Michelich CR, Dewhirst MW, Yuan F, Chilkoti A. Tumor vascular permeability, accumulation, and penetration of macromolecular drug carriers. Journal of the National Cancer Institute. 2006; 98:335-44. [PubMed: 16507830]

35. Yuan F, Leunig M, Huang SK, Berk DA, Papahadjopoulos D, Jain RK. Mirovascular Permeability and Interstitial Penetration of Sterically Stabilized ( Stealth ) Liposomes in a Human Tumor Xenograft. Cancer Research. 1994:3352-3356. [PubMed: 8012948]

36. Dewhirst MW, Shan S, Cao Y, Moeller B, Yuan F, Li CY. Intravital, fluorescence facilitates measurement of multiple physiologic functions and gene expression in tumors of live animals. Disease Markers. 2002; 18:293-311. [PubMed: 14646042]

37. Michel CC, Curry FE. Microvascular permeability. Physiological Reviews. 1999; 79:703-61. [PubMed: 10390517]

38. Pries AR, Neuhaus D, Gaehtgens P. Blood viscosity in tube flow : dependence on diameter and hematocrit American Journal of Physiology. Heart and Circulatory Physiology. 1992:1770-1778.

39. Dehouck B, Fenart L, Dehouck MP, Pierce A, Torpier G, Cecchelli R. A new function for the LDL receptor: transcytosis of LDL across the blood-brain barrier. Journal of Cell Biology. 1997; 138:877-889. [PubMed: 9265653]

40. Roberts RL, Fine RE. a Sandra, Receptor-mediated endocytosis of transferrin at the blood-brain barrier. Journal of Cell Science. 1993; 104(Pt 2):521-32. [PubMed: 8505377]

41. Burgess A, Ayala-Grosso CA, Ganguly M, Jordão JF, Aubert I, Hynynen K. Targeted delivery of neural stem cells to the brain using MRI-guided focused ultrasound to disrupt the blood-brain barrier. PloS One. 2011; 6:e27877. [PubMed: 22114718]

42. Alkins R, Burgess A, Ganguly M, Francia G, Kerbel R, Wels WS, et al. Focused ultrasound delivers targeted immune cells to metastatic brain tumors. Cancer Research. 2013; 73:1892-9. [PubMed: 23302230]

43. McDannold N, Vykhodtseva N, Hynynen K. Blood-brain barrier disruption, induced by focused ultrasound and circulating preformed microbubbles appears to be characterized by the mechanical index. Ultrasound in Medicine \& Biology. 2008; 34:834-40. [PubMed: 18207311]

44. Burgess A, Cho EE, Shaffaf L, Nhan T, Poon C, Hynynen K. The use of, two-photon microscopy to study the biological effects of focused ultrasound on the brain. Proc SPIE. 2012:822642822647 . 
45. Deng CX, Sieling F, Pan H, Cui J. Ultrasound-induced cell membrane porosity. Ultrasound in Medicine \& Biology. 2004; 30:519-26. [PubMed: 15121254]

46. Sheikov N, McDannold N, Vykhodtseva N, Jolesz F, Hynynen K. Cellularmechanisms of the blood-brain barrier opening induced by ultrasound in presence of microbubbles. Ultrasound in Medicine \& Biology. 2004; 30:979-89. [PubMed: 15313330] 
A)

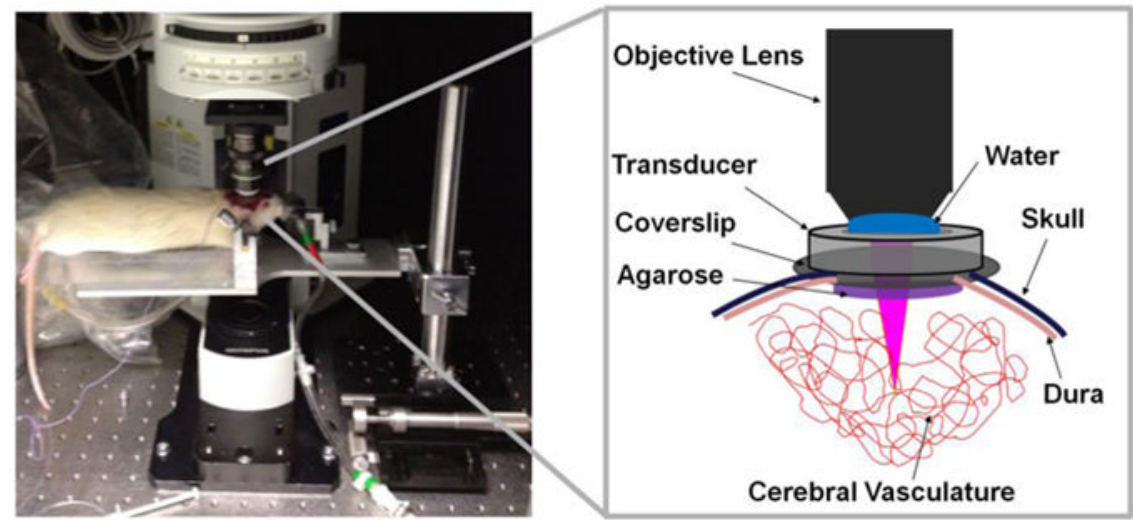

B)

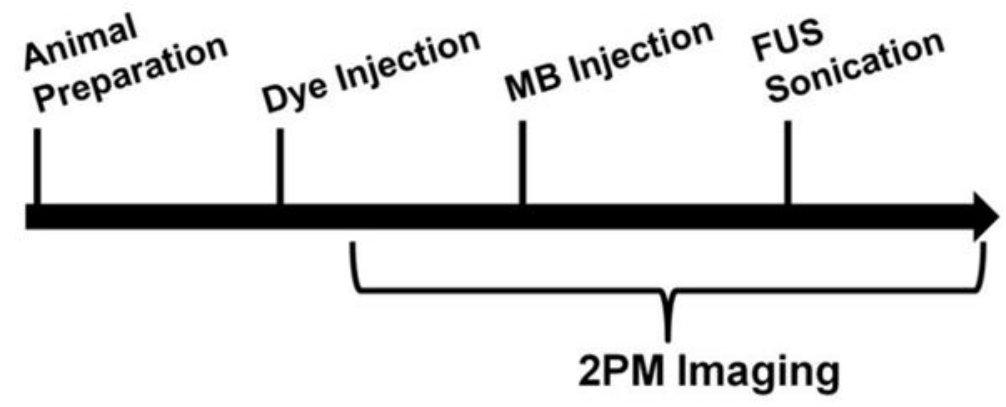

C)

Scan Interval (minutes)
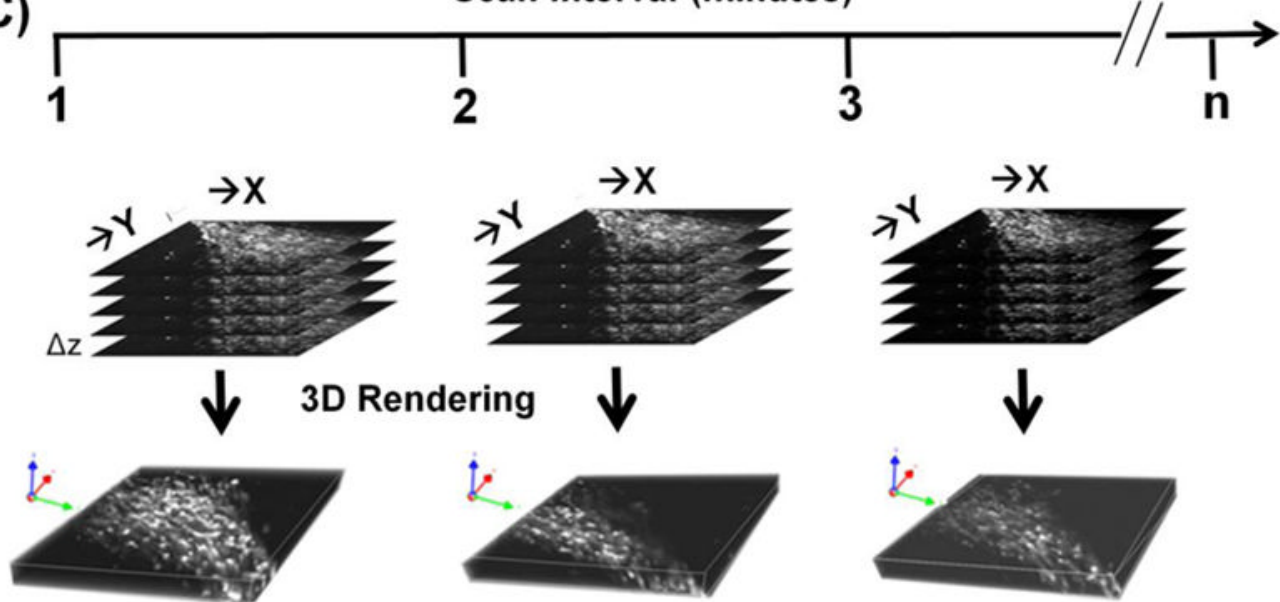

3D Rendering
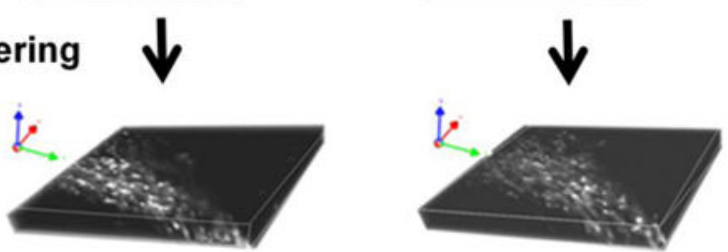

Figure 1. In vivo BBBD induced by FUS and monitored by $2 \mathrm{PFM}$ imaging

A) Schematic of the experimental setup used to induce BBBD in rats via MBs \& FUS. Ring configuration of the transducer also facilitates optical transparency for 2PFM imaging. B) Experimental timeline. C) 4D XYZT acquisition of 2PFM imaging. 
A)
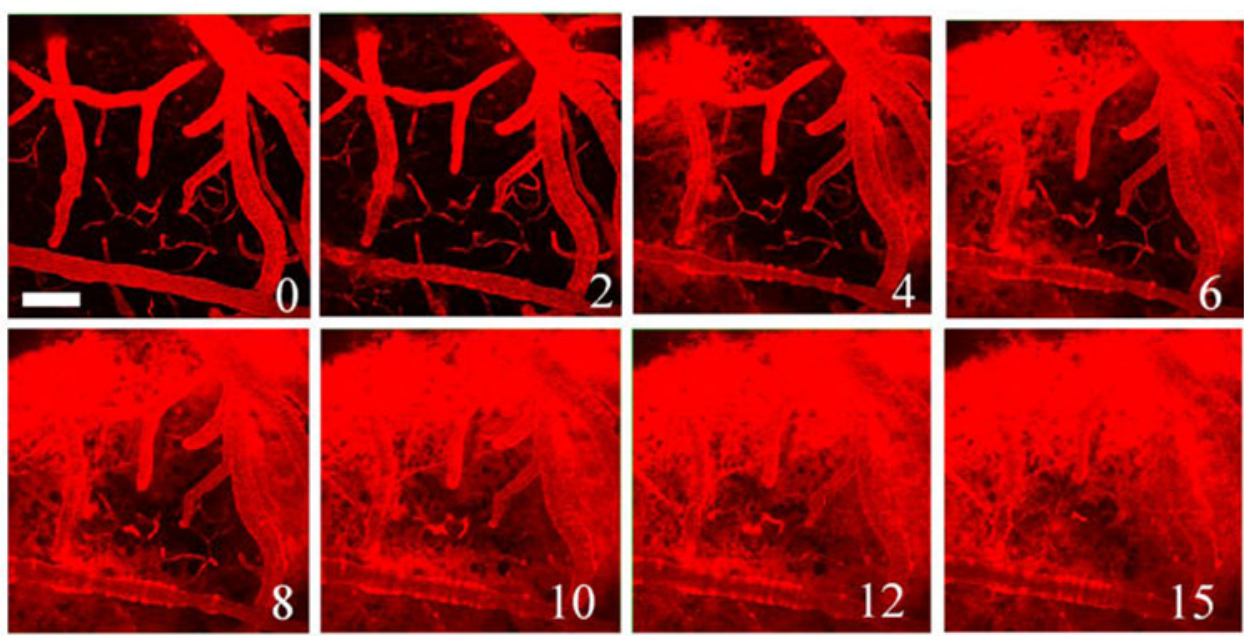

B)

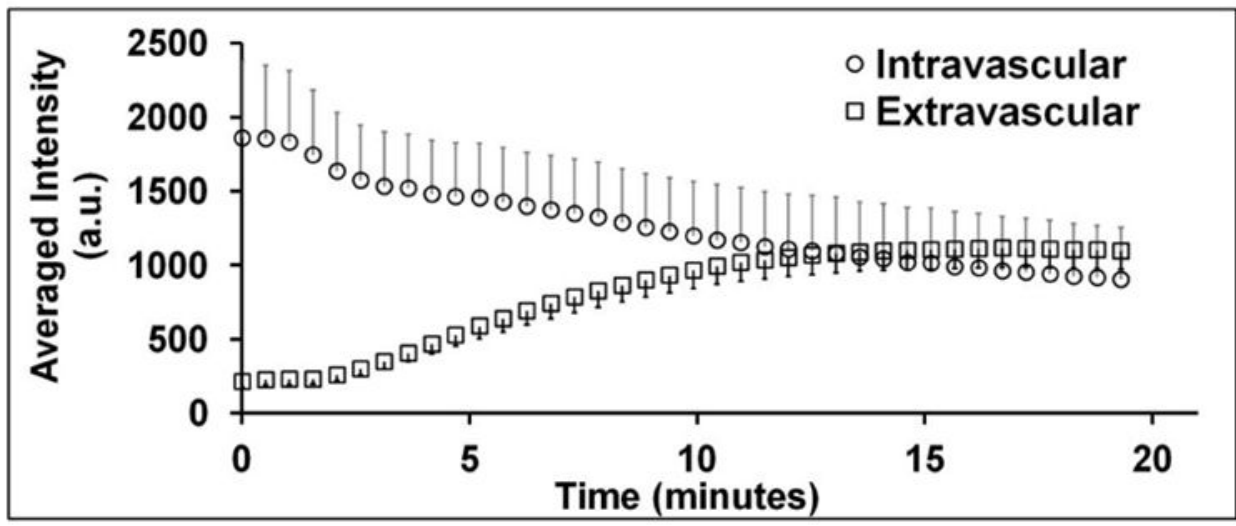

C)

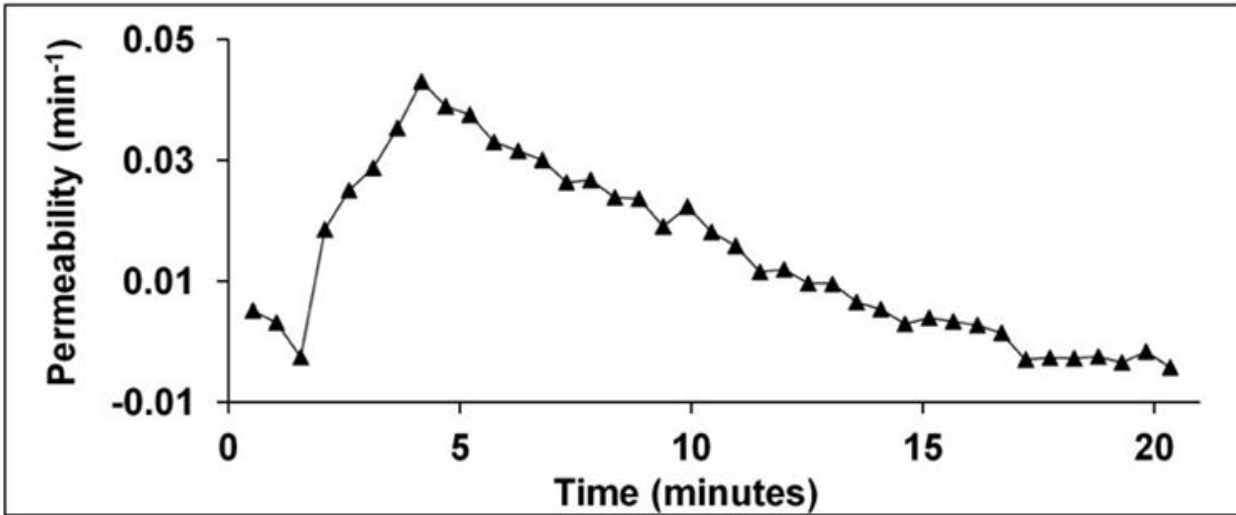

Figure 2. Data analysis of 2PFM data capturing fluorescent dye leakage upon BBBD

A) Depth projection images illustrate the transient BBBD induced by MBs \& FUS at 0.6 MPa (scale bar: $100 \mu \mathrm{m})$. Sonication and MB injection occurred during the first 2 minutes while the vessels remained impermeable to dextran conjugated Texas Red TR10kDa. As soon as sonication ceased, disruption started at multiple vessels within the imaging FOV and extravascular signal increases over time. B) Quantitative measurement of averaged fluorescent signal intensities associated with intravascular and extravascular compartment over time. C) Permeability was evaluated accordingly. 
A)

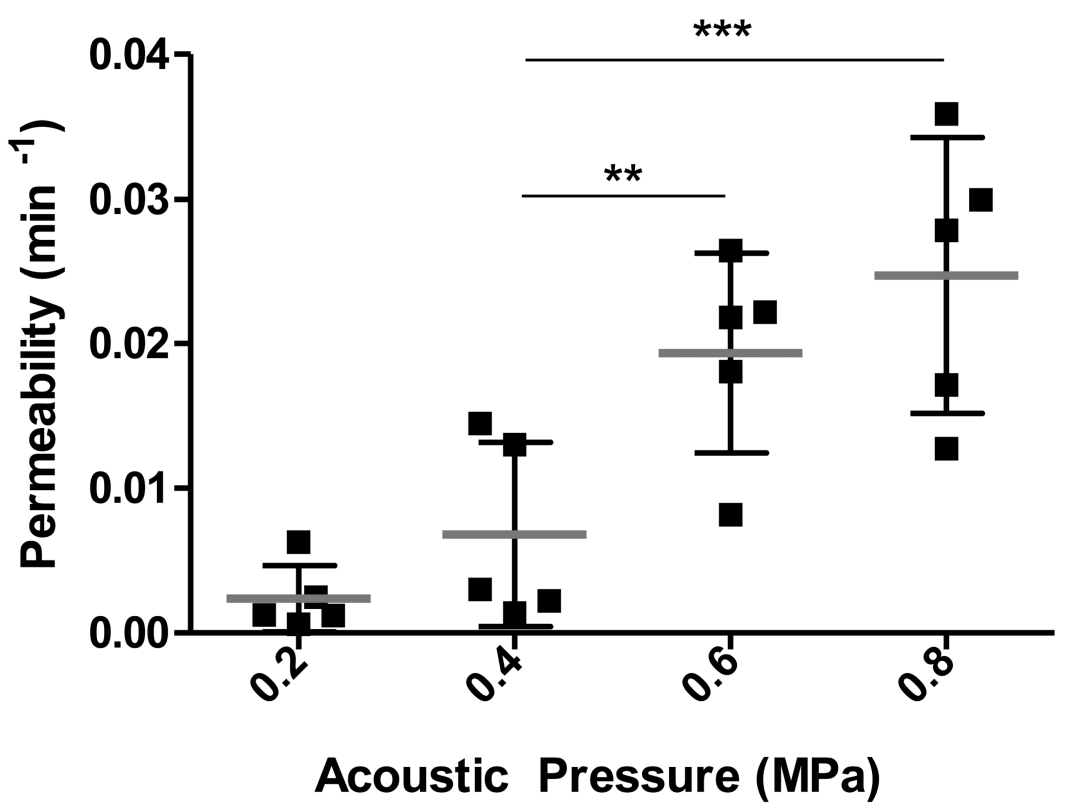

B)

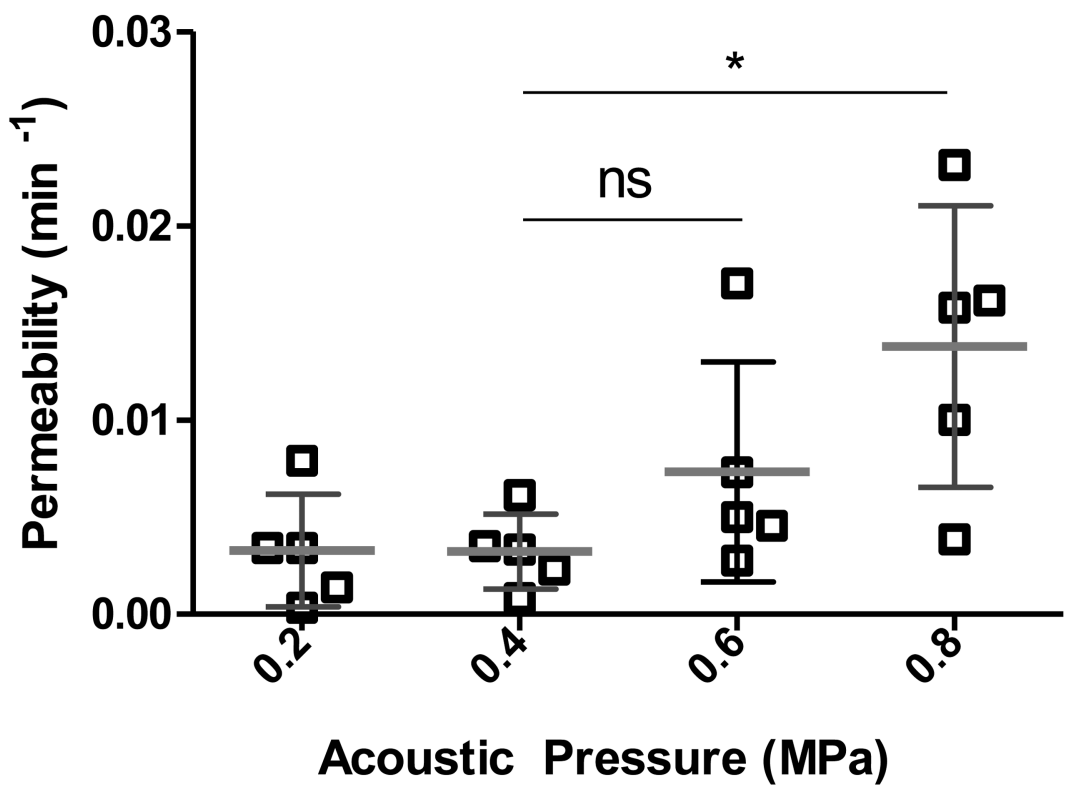

Figure 3. Effect of acoustic pressure on permeability dextran conjugated Texas Red across the BBB

A) Measured for all 20 cases of TR10kDa delivered across the BBB ( $n=5$ per pressure). Two-way ANOVA in combination with Bonferroni post-tests confirm statistical significance in permeability induced at low pressure of $0.4 \mathrm{MPa}$ with respect to the higher acoustic pressure of 0.6 MPa and 0.8 MPa. B) Measured permeabilities for all 20 cases of TR70kDa delivered across the BBB ( $\mathrm{n}=5$ per pressure). Two-way ANOVA in combination with Bonferroni post-tests suggest there is no statistical significance in permeabilities between the $0.4 \mathrm{MPa}$ and 0.6 $\mathrm{MPa}$, whereas statistical significance was found for permeabilities induced at $0.4 \mathrm{MPa}$ and $0.8 \mathrm{MPa}$. 
A)

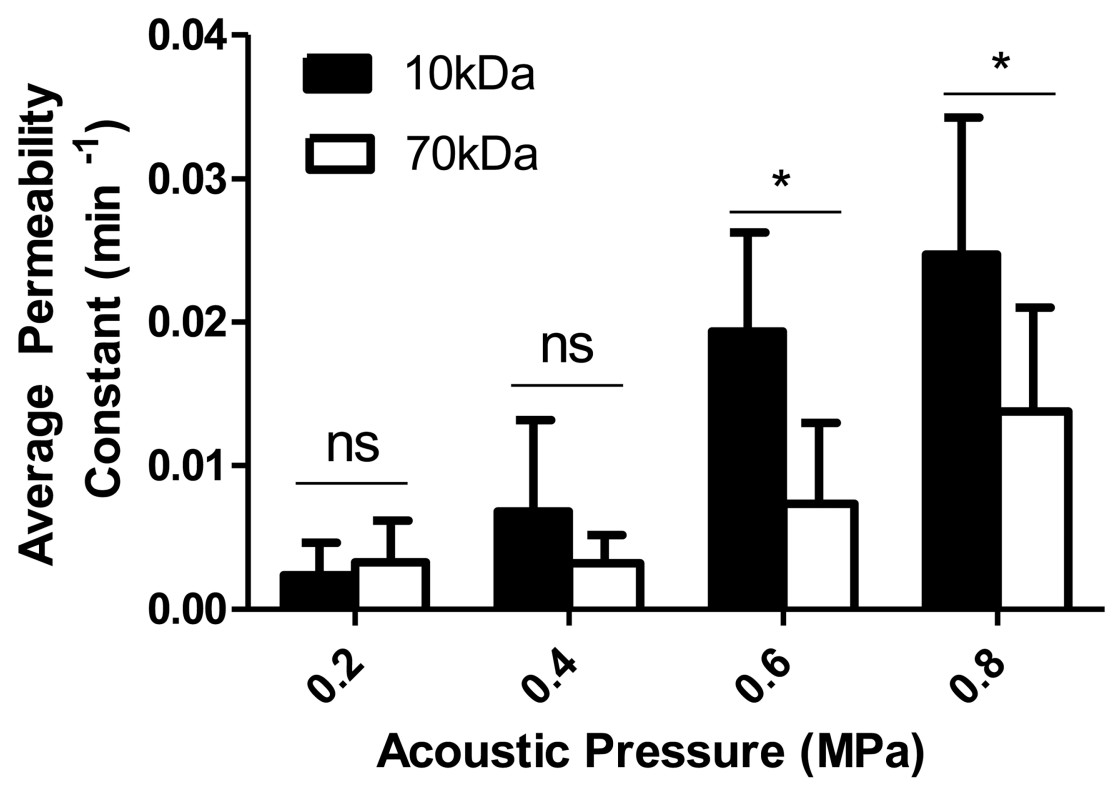

B)

$\mathbb{M} 10 \mathrm{kDa} \square 70 \mathrm{kDa}$

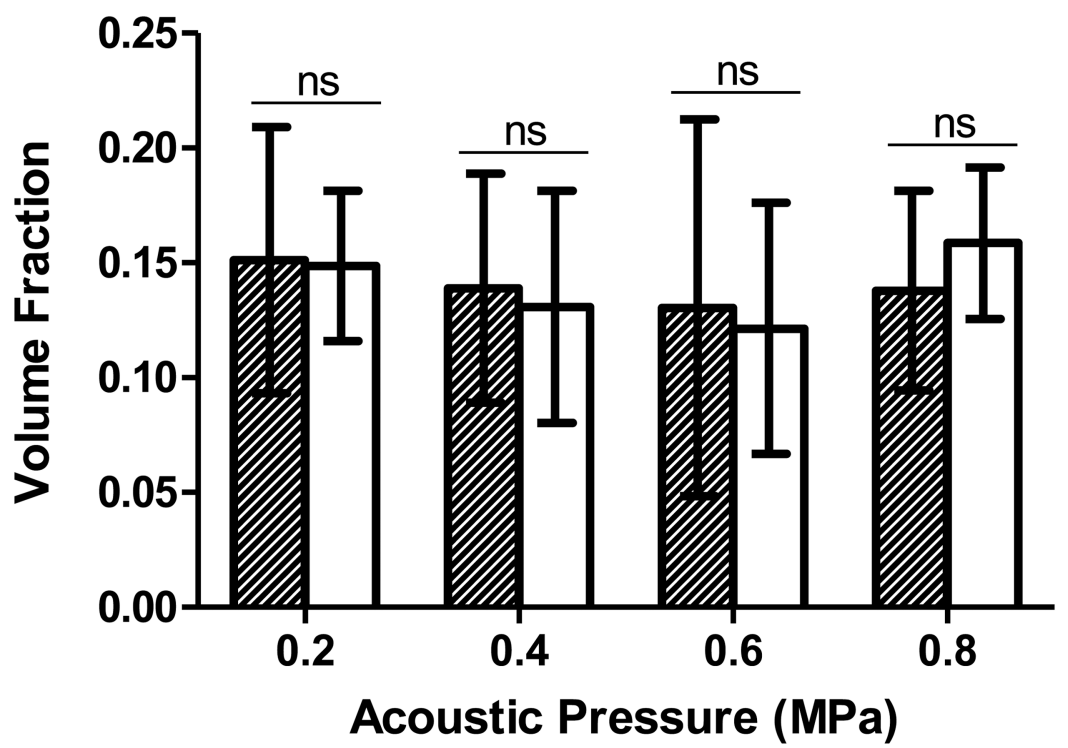

Figure 4. Effect of substance size on enhanced BBB permeability

A) At each pressure, average permeability constant $(n=5)$ was compared between TR10kDa and TR70kDa. Two-way ANOVA in combination with Bonferroni post-tests were performed as multiple comparisons. The $\mathrm{p}$ values at high pressure $(0.6 \mathrm{MPa}$ and $0.8 \mathrm{MPa})$ are statistically significant, elucidating different level of permeability enhancement between two MWs. B) At each pressure, average volume fraction $(n=5)$ was compared between TR10kDa and TR70kDa. Two-way ANOVA in combination with Bonferroni post-tests were performed as multiple comparisons and confirmed there was no statistical significant between each MW pair across all 4 acoustic pressures. 

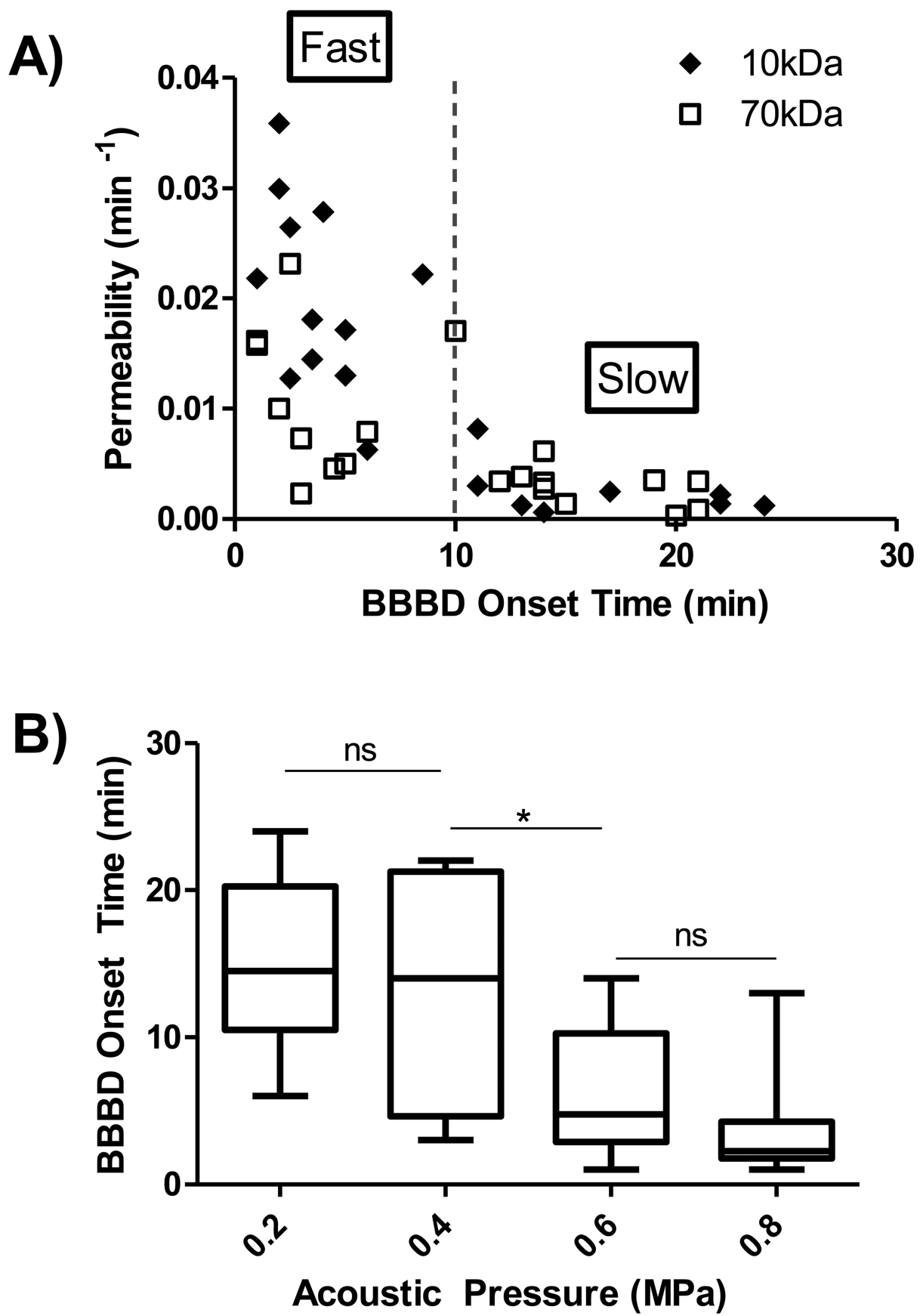

Figure 5. BBBD onset in relation to permeability and acoustic pressure A) Inverse relationship between BBBD onset and permeability. B) Inverse relationship between BBBD onset and acoustic pressure. One-way ANOVA followed by Bonferroni's Multiple Comparison Test confirms a statistical significance in BBBD onset between 0.4 $\mathrm{MPa}$ and 0.6 MPa. 
A)

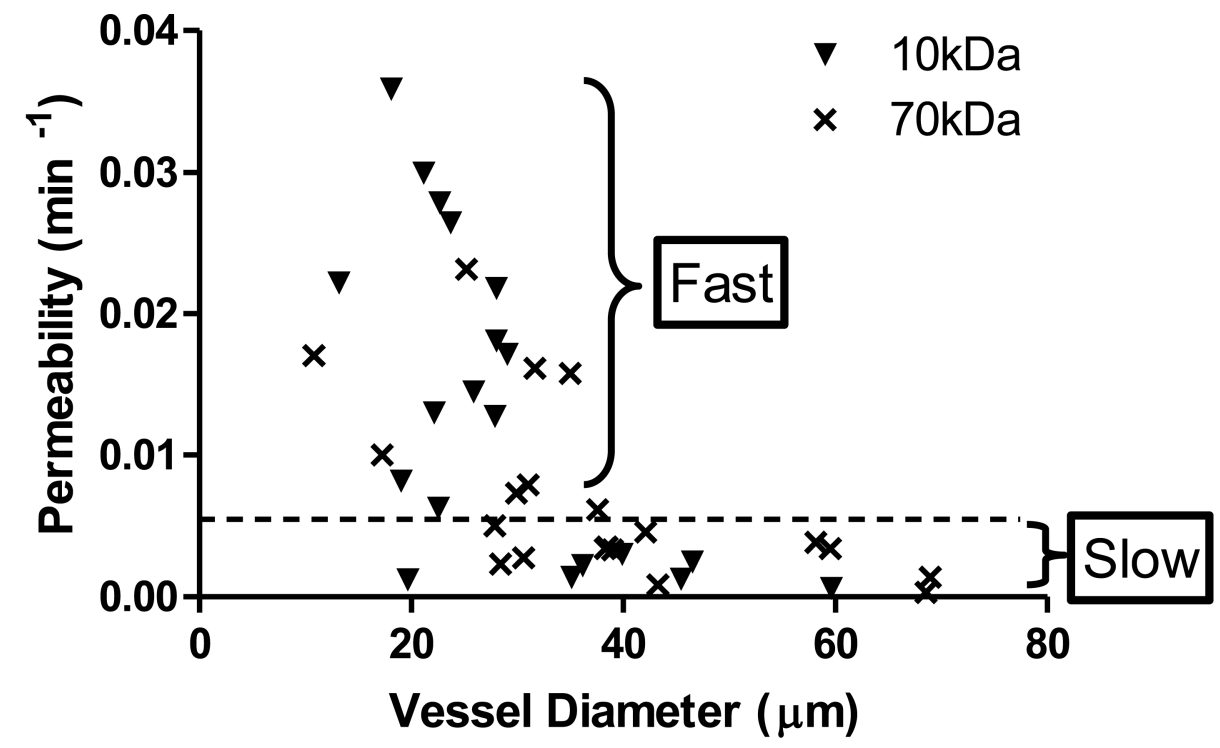

B)

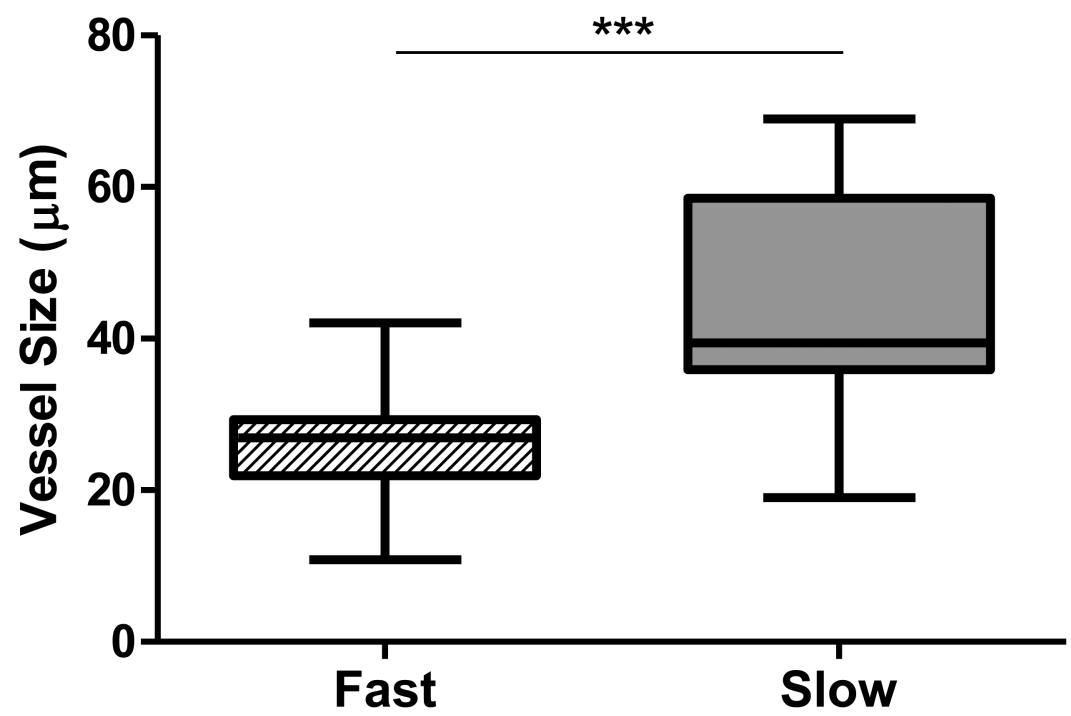

Figure 6. Effect of vessel diameter on enhanced BBB permeability

A) Vessel size distribution in correlation with permeability constant: large vessels (20-70 $\mu \mathrm{m})$ are prone to slow leakage kinetics and low permeability; whereas smaller vessels $(10-30 \mu \mathrm{m})$ are subjected to fast leakage kinetics and high permeability. B) Statistical analysis (two-tailed t test) indicates significant difference $(p<0.0001)$ in vessel size responsible for fast and slow leakage types. 